\title{
The Optical Properties Test on Shading Fabric
}

\author{
Peng Yue ${ }^{1, a}$, Qinlian Tao ${ }^{1, b}$
}

${ }^{1}$ Building Water and Energy Conservation Inspection Department, Shanghai Jianke Technical

Assessment of Construction Co., Ltd, 201108, China

ayuepeng@jktac.com, btql@ jktac.com

Keywords: Shading fabric, Optical properties, Shading coefficient, measuring methods

\begin{abstract}
In order to study the heat insulation and indoor light adjusting ability of fabric, the optical properties of usual shading fabric were tested using spectrophotometer which measurement range is $190 \mathrm{~nm} \sim 3300 \mathrm{~nm}$. Under different open porosity, the ultraviolet transmittance, solar direct transmittance, solar direct reflectance, visible light transmittance, shading coefficient of black and white fabric which has different position with glazing were tested, and fabric itself shading coefficient calculation method was put forward. Test results show that: the shading effect difference between plain and twill fabric was small. With the increase of porosity of fabric, shading effect decreased. External shading fabric has the better shading effect than intermediate fabric, and internal shading fabric is the worst. The fabric color has a great influence on the optical properties, from the point of energy conservation, dark fabric is the best choice using in external shading, but on intermediate and internal shading light fabric has priority.
\end{abstract}

\section{Introduction}

Recently, as people pay more attention to indoor comfort and energy saving, people prefer use professional shading blinds instead of ordinary curtain for its heat insulation and adjustment of sunlight distribution. Shading has significant effect to reduce building energy consumption and improve the indoor living comfort. As a kind of effective building energy saving technology, shading is strongly supported by government. Many enterprises seized this opportunity and produced many kinds of indoor and outdoor shading blinds [1]. Since March 1, 2014, China carried out JG/T 423-2013 "Architectural shading membrane" and JG/T 424-2013 "General requirements for solar shading fabric of building" to require the optical properties of shading fabric, which include ultraviolet transmittance, solar direct transmittance, solar direct reflectance, visible light transmittance and shading coefficient.

In the fabric optical properties field, Hangyan Zhu of Donghua Textile Institute [2], Biling Yang of Guangdong textile department of Career Technical College [3] had researched the main factors that affect the anti-ultraviolet performance of textiles, such as fiber materials, yarn structure, fabric construction parameters. Baoguo Zhang of Northwest Institute of Textile Science and Technology [4] had tested the infrared reflectance properties of varies fabric which have different materials and structure, but only limited to a single test of ultraviolet light, visible light or infrared spectroscopy. The test instrument was time consuming and imprecise. While evaluate optical performance of functional fabrics, people prefer to test the reflectance, absorption and transmission properties in the solar spectrum range [4]. Shading Coefficient is ratio of the solar factor of the glass to the solar factor of a reference glass, it not only including the solar direct transmittance into the room, but also including secondary internal heat transfer factor. Shading coefficient can well represent the optical properties of shading fabric.

In order to provide the reference for the application and performance evaluation of shading 
fabric, the optical properties of usual shading fabric were tested by Shanghai Jianke Technical Assessment of Construction Co., Ltd using spectrophotometer which measurement range is 190nm 3300nm. Under different open porosities, the ultraviolet transmittance, solar direct transmittance, solar direct reflectance, visible light transmittance, shading coefficient of black and white fabric which has different situation with window were tested, and fabric itself shading coefficient calculation method was put forward.

\section{Test Instrument, Principle and Sample}

Lambda 950 produced by Perkin Elmer Co., Ltd., with double beam and double monochromatic system ratio type spectrophotometer, assembling a pre calibration and automatic switching of tungsten and deuterium lamp and high sensitivity photoelectric multiplier tube receiver. Due to the fabric surface is not flat, so integrating sphere is used to test the optical transmission and reflectance of the fabric to collect diffuse and scattered light in all direction. Scan wavelength range is 220 $2500 \mathrm{~nm}$. The scanning wavelength interval is $5 \mathrm{~nm}$. The scanning speed is $700 \mathrm{~nm}$ per min. The measurement can test the light reflectance and transmission properties of textiles in the wide spectral range, with high speed and high precision.

According to China JG/T 356-2012 "Test methods of building shading product on indoor environment thermal and visual comfort", In the solar spectrum range, using spectrophotometer, Through the ratio of textile layer's transmission luminous flux to air with the same thickness, the ratio of reflectance luminous flux to reference board, and then use the standard solar radiation relative spectral distribution of illuminant, optical transmission and reflectance of the fabric can be calculated. Such as the calculation of visible light transmittance can be export:

$$
\tau_{v}=\frac{\int_{380}^{780} D_{\lambda} \tau(\lambda) V(\lambda) d \lambda}{\int_{380}^{780} D_{\lambda} V(\lambda) d \lambda} \approx \frac{\sum_{\lambda=380}^{780} D_{\lambda} \tau(\lambda) V(\lambda) \Delta \lambda}{\sum_{\lambda=380}^{780} D_{\lambda} V(\lambda) \Delta \lambda}
$$

where, $\tau_{v}$ is visible light transmittance, $D_{\lambda}$ is relative spectral distribution of illuminant D65, $\tau(\lambda)$ is spectral transmittance, $V(\lambda)$ is spectral luminous efficiency, $\Delta \lambda$ is wavelength interval. Other optical parameters can be got in the same way, the ultraviolet wavelength range is $280 \sim 380 \mathrm{~nm}$, the visible wavelength range is $380 \sim 780 \mathrm{~nm}$, the solar wavelength is $350 \sim 1800 \mathrm{~nm}$.

According to Chinese JG/T 356-2012,shading coefficient of shading fabric defined as the ratio of the total solar energy transmittance of solar protection devices combined with glazing to glazing. So while the different relative position between solar protection devices and glazing, the different total solar energy transmittance, and shading fabric has different shading coefficient. The smaller shading coefficient indicates the better shading effect.

For External solar protection device, the total solar energy transmittance for glazing and an external solar protection device is given by:

$$
g_{\text {tot }}=\tau_{s} \cdot g+\alpha_{s} \cdot \frac{\Lambda}{\Lambda_{2}}+\tau_{s} \cdot(1-g) \frac{\Lambda}{\Lambda_{1}}
$$

where,

$$
\Lambda=\frac{1}{\frac{1}{\Lambda_{1}}+\frac{1}{\Lambda_{2}}+\frac{1}{U_{w}}}
$$

For Internal solar protection device, the total solar energy transmittance for glazing and an 
external solar protection device is given by:

$$
g_{\text {tot }}=g \cdot\left(1-g \cdot \rho_{s}-\alpha_{s} \cdot \frac{\Lambda}{\Lambda_{2}}\right)
$$

where,

$$
\Lambda=\frac{1}{\frac{1}{\Lambda_{2}}+\frac{1}{U_{w}}}
$$

For integrated solar protection device, the total solar energy transmittance for glazing and an external solar protection device is given by:

$$
g_{\text {tot }}=\tau_{s} \cdot g+g\left[\alpha_{s}+(1-g) \rho_{s}\right] \frac{\Lambda}{\Lambda_{3}}
$$

where,

$$
\Lambda=\frac{1}{\frac{1}{\Lambda_{3}}+\frac{1}{U_{w}}}
$$

In formula (2)-(7), $\Lambda_{1}$ is thermal conductance for shading fabric, $6 \mathrm{~W} /\left(\mathrm{m}^{2} \cdot \mathrm{K}\right), \Lambda_{2}$ is thermal conductance for the air between shading fabric and glazing, $18 \mathrm{~W} /\left(\mathrm{m}^{2} \cdot \mathrm{K}\right), \Lambda_{3}$ is thermal conductance for shading fabric in integrated solar protection device, $3 \mathrm{~W} /\left(\mathrm{m}^{2} \cdot \mathrm{K}\right), U_{w}$ is thermal transmittance for glazing, $g$ is total solar energy transmittance for glazing, $\tau_{s}, \rho_{s}, \alpha_{s}$ are solar direct transmittance of the glazing, solar direct reflectance, solar direct absorptance. In order to facilitate the comparison, we use double clear glass window which $g$ is $0.75, U_{w}$ is $3.0 \mathrm{~W} /\left(\mathrm{m}^{2} \cdot \mathrm{K}\right)$.

According to the above calculation method, while comparing shading coefficient of different fabric, the different relative position between solar protection devices and glazing needed to put forward, and this shading coefficient cannot represent the fabric itself, Therefore, we need to increase testing method for fabric shading coefficient, Chinese GB/T 2680-1994 "Determination of light transmittance, solar direct transmittance, total solar energy transmittance and ultraviolet transmittance for glass in building and related glazing factors" is recommended to calculate shading coefficient of fabric, which the calculation process is simple and results is intuitive. The shading coefficient of fabric is given by:

$$
S C=\frac{g_{s}}{0.889}
$$

where, $g_{s}$ is total solar transmittance of the fabric which is equal to solar direct transmittance plus secondary internal heat transfer factor $q_{i}$, secondary internal heat transfer factor $q_{i}$ is given by:

$$
\begin{gathered}
q_{i}=\alpha_{s} \times \frac{h_{i}}{h_{i}+h_{e}} \\
h_{i}=3.6+\frac{4.4 \varepsilon_{i}}{0.83}
\end{gathered}
$$

where, $h_{e}, h_{i}$ are the heat transfer coefficients towards the outside and inside respectively, $h_{e}$ is 23 $\mathrm{W} /\left(\mathrm{m}^{2} \cdot \mathrm{K}\right), \varepsilon_{i}$ is the corrected emissivity of the inside surface. According to this calculation method, only solar direct transmittance and the corrected emissivity of the inside surface are needed to test for the shading coefficient of shading fabric, and this shading coefficient regardless of the optical properties and position of the window, is an inherent property of the fabric itself.

The most commonly used high performance polyester plain and twill fabric are selected for the test, which thickness is $0.4 \mathrm{~mm}$, the open porosity is $1 \%, 3 \%, 5 \%, 10 \%$ respectively. All the samples 
are humidifying treatment under the condition of 20 centigrade and $65 \%$ relative humidity. Fig. 1 and Fig. 2 are the structure of black and white twill weave fabric for open porosity of $5 \%$.

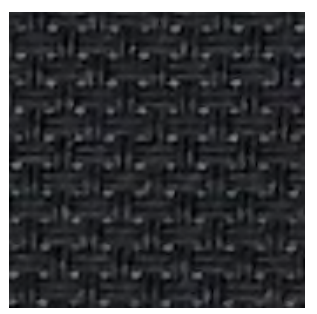

Fig. 1 Black twill weave fabric with open porosity of $5 \%$.

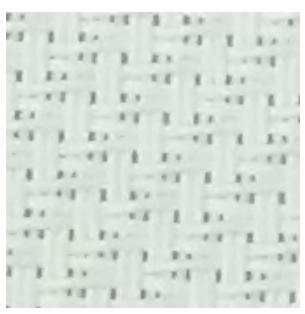

Fig. 2 White twill weave fabric with open porosity of $5 \%$.

\section{Test Results}

According to the calculation principle, the optical properties of fabrics are got by using Spectrophotometer Lambda950. Fig. 3 and Fig. 4 are transmittance and reflectance spectrum curve respectively from ultraviolet band to near-infrared band. The optical properties of fabric in the ultraviolet, visible and near-infrared bands on the transmittance and reflectance can be clearly observed. Fig. 3 and Fig. 4 show that the white fabric has low ultraviolet transmittance, and the transmittance of visible light band increased gradually. The solar direct transmittance and reflectance of white fabric has a larger percentage than black fabric, and the light is selective transmitted and reflected, while direct absorptance in the solar spectrum of black fabric accounted for a large percentage.

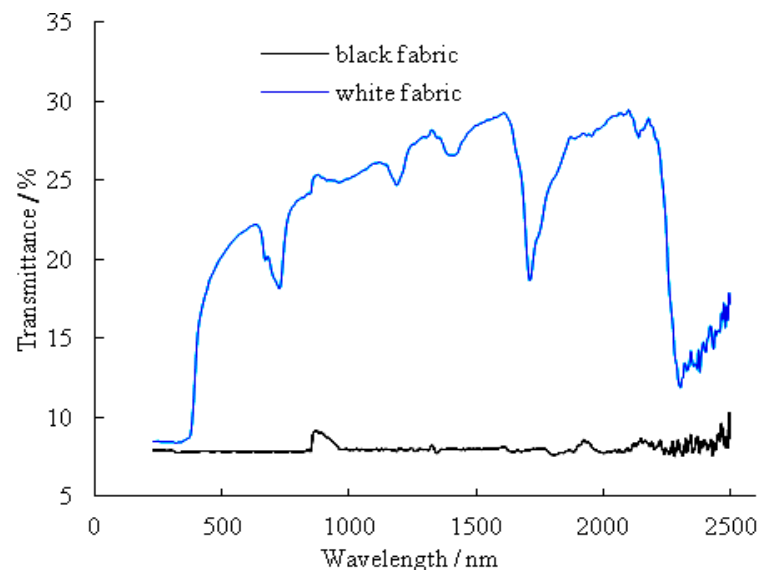

Fig. 3 Transmittance spectrum curve of fabric with open porosity of $5 \%$.

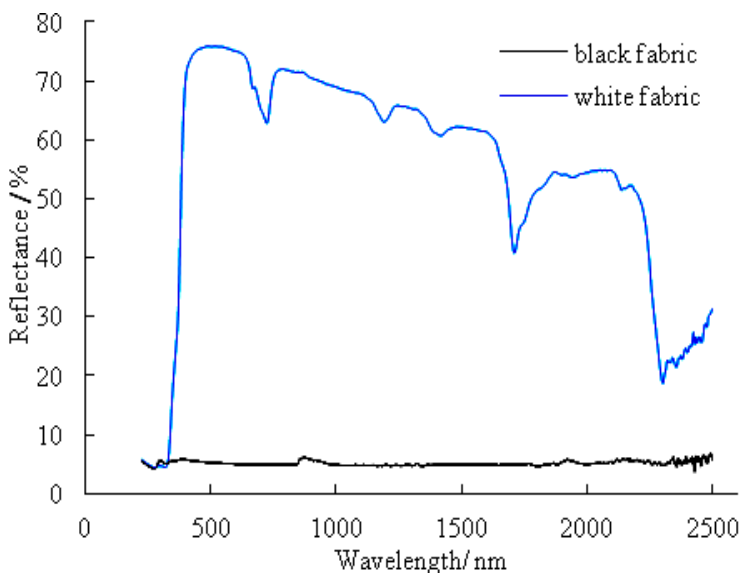

Fig. 4 Reflectance spectrum curve of fabric with open porosity of $5 \%$.

For the quantitative analysis of the optical properties of shading fabric, test results are expressed in Table 1 and Table 2. Table 1 is the optical performance of plain fabric, Table 2 is the optical properties of twill fabric. Where $\tau_{u v}, \tau_{v}$ are ultraviolet transmittance, visible light transmittance respectively. $S C_{W}, S C_{N}, S C_{Z}$ are shading coefficients of external, Internal, integrated shading fabric respectively according to Chinese JG/T 356-2012. SC is fabric shading coefficient according to Chinese GB/T 2680-1994. 
Table 1. The optical performance of plain fabric.

\begin{tabular}{cccccccccccc}
\hline NO. & color & open porosity / \% & $\tau_{u v}$ & $\tau_{s}$ & $\rho_{s}$ & $\alpha_{s}$ & $\tau_{v}$ & $S C_{W}$ & $S C_{N}$ & $S C_{Z}$ & $S C$ \\
\hline 1 & white & 1 & 2.3 & 13.4 & 74.0 & 12.6 & 12.7 & 0.16 & 0.43 & 0.29 & 0.19 \\
2 & white & 3 & 3.7 & 14.9 & 73.4 & 11.7 & 13.9 & 0.18 & 0.43 & 0.30 & 0.21 \\
3 & white & 5 & 8.7 & 22.1 & 67.5 & 10.4 & 21.3 & 0.26 & 0.48 & 0.36 & 0.29 \\
4 & white & 10 & 11.4 & 28.5 & 61.4 & 10.1 & 26.9 & 0.33 & 0.52 & 0.41 & 0.36 \\
5 & black & 1 & 1.5 & 1.6 & 5.2 & 93.2 & 1.5 & 0.14 & 0.83 & 0.49 & 0.31 \\
6 & black & 3 & 1.8 & 2.0 & 5.5 & 92.5 & 1.8 & 0.15 & 0.83 & 0.49 & 0.32 \\
7 & black & 5 & 7.8 & 7.9 & 5.2 & 86.9 & 7.8 & 0.20 & 0.84 & 0.52 & 0.37 \\
8 & black & 10 & 13.7 & 13.8 & 4.9 & 81.3 & 13.8 & 0.26 & 0.85 & 0.55 & 0.41 \\
\hline & \multicolumn{7}{c}{ Table 2. The optical properties of twill fabric. } & & & \\
\hline NO. & color & open porosity /\% & $\tau_{u v}$ & $\tau_{s}$ & $\rho_{s}$ & $\alpha_{s}$ & $\tau_{v}$ & $S C_{W}$ & $S C_{N}$ & $S C_{Z}$ & $S C$ \\
\hline 9 & white & 1 & 1.2 & 12.2 & 74.4 & 13.4 & 8.7 & 0.15 & 0.42 & 0.28 & 0.18 \\
10 & white & 3 & 4.0 & 17.6 & 70.7 & 11.7 & 17.2 & 0.21 & 0.45 & 0.32 & 0.24 \\
11 & white & 5 & 5.5 & 19.5 & 66.4 & 14.1 & 18.5 & 0.23 & 0.48 & 0.35 & 0.27 \\
12 & black & 1 & 1.2 & 1.2 & 3.8 & 95.0 & 1.2 & 0.14 & 0.84 & 0.49 & 0.32 \\
13 & black & 3 & 2.5 & 2.6 & 5.0 & 92.4 & 2.5 & 0.15 & 0.83 & 0.49 & 0.33 \\
14 & black & 5 & 3.3 & 3.5 & 6.5 & 90.0 & 3.3 & 0.16 & 0.82 & 0.49 & 0.33 \\
\hline
\end{tabular}

\section{Analysis of Test Results}

Form Table 1 and Table 2, the following results are given:

a) With the increase of porosity of fabric, the ultraviolet transmittance, solar direct transmittance, visible light transmittance and shading coefficient increased, while the solar direct reflectance and solar direct absorptance decreased.

b) The shading effect difference between plain and twill fabric was small, while used in the same position, the largest shading coefficient different between twill fabric and plain fabric with the same open porosity is 0.03 .

c) External shading fabric has the better shading effect than integrated shading fabric, and internal shading fabric effect is the worst. Such as No.14 shading fabric, when used for external shading, internal shading, integrated shading device, shading coefficients are $0.16 、 0.82 、 0.49$ respectively, it is easily confusing and illegal used.

d) When used for external shading, shading coefficient of white fabric is slightly larger than the black fabric and difference is in the range of 0.1, but when used for internal shading, the black fabric has the worse shading effect than white fabric, shading coefficient of white fabric is 0.42 to 0.52 , shading coefficient of black fabric is 0.82 to 0.8 , when used for integrated shading, the black fabric also has the worse shading effect than white fabric obviously, shading coefficient of white fabric is 0.28 to 0.41 , shading coefficient of black fabric is 0.49 to 0.55 .

e) Black fabric has play a contributing role in shading, but because of the large percentage of solar energy absorptance would be changed into the cold load through thermal transfer. It would increase the building energy consuming comparing to white fabric. At the same time, black fabric prevent larger percentage visible light into the interior than white fabric. For example, visible light transmittances of white plain fabric with $5 \%$ open porosity is $21.3 \%$, but on the contrary the black fabric is $7.8 \%$, it would lead to the increasing of indoor lamplight and consume additional electric. 


\section{Conclusion}

a) The shading effect difference between plain and twill fabric was small, through the adjustment of open porosity of the shading fabric can meet the demands of different building shading.

b) External shading fabric has the better shading effect than integrated shading fabric, and shading effect of internal shading fabric is the worst.

c) Dark fabric is a good choice for external shading, but when used in internal shading and integrated shading, light color fabric will be better.

d) Shading coefficient of fabric in Chinese JG/T 356-2012 is a changeable parameter with the different position related to glazing, but shading coefficient in Chinese GB/T 2680-1994 is constant and easy to distinguish.

\section{References}

[1] F. X. Tu, Z. S. Zhang, J. D. Cai, Structure and Application of Interior and Exterior Sunshade Curtain. Archit. Technol. 12 (2013) 1095-1098.

[2] H. G. Zhu, W. D. Yu, The Performance and Evaluation of Anti Ultraviolet Textiles, China Textile Leader. 5 (2003) 141-143.

[3] B. L. Yang, X. P. Luo, Factors Affecting Anti Ultraviolet Performance of Textiles and Related Tests, .China Textile Leader. 04 (2014) 88-92.

[4] B. G. Zhang, W. L. Xu, T. W. Shi, Study on Fabric Performance of Infrared Reflectance. J. Northwest Insit. Textile Sci. Technol. 6 (2001) 84-93.

[5] X. S. Liao, L. Q. Zhang, B. Y. Chen. Research Status and Development Trend of Textile Optical Performance Measurement, Adv. Textile Technol. 2 (2009) 66-68. 